\title{
Nestin as a Marker for Proliferative Endothelium in Gliomas
}

\author{
Ken-ichi Sugawara, Hideyuki Kurihara, Mitsuko Negishi, Nobuhito Saito, \\ Yoichi Nakazato, Tomio Sasaki, and Toshiyuki Takeuchi \\ Department of Molecular Medicine (KS, MN, TT), Institute for Molecular and Cellular Regulation, Gunma University, \\ and Departments of Neurosurgery (KS, HK, NS, TS) and Pathology (YN), Gunma University School of Medicine, \\ Maebashi 371-8512, Japan
}

\begin{abstract}
SUMMARY: Nestin is one of the intermediate filaments abundantly produced in the developing central nervous system and somites in the embryonic stage. Nestin is also reportedly detected in gliomas/glioblastomas. We retested nestin expression in brain tumors having a range of malignancy grades using immunostaining. The intensity of nestin immunostaining roughly paralleled the malignancy grade of the gliomas. However, many tumors were negative for nestin immunostaining, while nestin immunostaining was invariably detected in tumor endothelium regardless of glioma malignancy grades or brain tumor types. We suspected that angiogenic epithelial cells may express nestin, and we found that nestin was highly positive in bovine aortic endothelial cells in static culture. However, nestin expression decreased when the endothelial cells underwent laminar shear stress flow, under which endothelial cells exhibit differentiated features and a decreased rate of growth. Because nestin is highly expressed in growing endothelial cells, we examined its expression in hemangioblastomas because hemangioblasts are thought to be a precursor for angiogenic epithelial cells. As expected, nestin immunostained strongly in all four samples of hemangioblastomas. We suggest that nestin is not only a marker for neuroepithelial stem cells and glioma cells but also for tumor endothelial cells during rapid growth. (Lab Invest 2002, 82:345-351).
\end{abstract}

$N$ estin is one of the intermediate filaments, together with vimentin and glial fibrillary acidic protein (GFAP), and is detected abundantly in neuroepithelial stem/progenitor cells in the growing central nervous system of embryonal rats and humans (Lendahl et al, 1990; Messam et al, 2000; Tohyama et al, 1992, 1993). Nestin forms intermediate filament bundles, perhaps with vimentin, by copolymerization in neuroepithelial cells (Eliasson et al, 1999; Rutka et al, 1999). Nestin mRNA is expressed highly in the cerebrum of the developing rat embryo at embryonic day 15 (E15), declines toward postnatal day 12 (P12), and disappears from P18 to the adult stage (Lendahl et al, 1990). Using nestin transgene-promoted $\beta$-galactosidase expression analysis in mice, LacZ activity has been detected in the neuroepithelium and somites shortly after neural tube closure (E9) (Zimmerman et al, 1994). The LacZ staining becomes stronger in the proliferative ventricular zones of the mouse embryonic striatum and cerebral cortex at E14.5 and E16.5 and decreases in expression in the adult cortex, becoming restricted to a population of ependymal cells. Substantial

Received December 12, 2001.

This work is supported by grants-in-aid from the Ministry of Education, Science, Sports, and Culture.

Address reprint requests to: Dr. Toshiyuki Takeuchi, Department of Molecular Medicine, Institute for Molecular and Cellular Regulation, Gunma University, Showa-machi, Maebashi 371-8512, Japan. E-mail: tstake@showa.gunma-u.ac.jp nestin has also been detected in human gliomas and glioblastomas (Dahlstrand et al, 1992). Nestin immunostaining has frequently been observed in highly malignant gliomas, especially glioblastomas, as compared with the less malignant forms such as pilocytic astrocytomas. In contrast, nestin is rarely detected by immunostaining in nonneoplastic brain tissues, occurring sometimes faintly in vascular endothelial cells.

Nestin mRNA is approximately 6.2 kilobases long, and its gene contains three introns. Interestingly, neuroepithelium-specific nestin expression is driven by the second intron of the nestin gene, whereas muscle precursor-specific expression is driven by the first intron (Lothian and Lendahl, 1997; Zimmerman et al, 1994). We previously examined nestin expression in seven human glioma/glioblastoma-derived culture cell lines (Kurihara et al, 2000). The level of expression varied from high (U251, KG-1C) to nondetectable (NP-2, LN-Z308, T98G) according to Northern blot analysis. The expression levels did not parallel the growth rates of the cell lines, although the degree of malignancy generally reflects tumor doubling time in vivo. The neuronal cell-specific regulator, consisting of the second intron before the $5^{\prime}$ upstream region of the gene, drove LacZ expression in parallel with the extent of mRNA expression in each cell line (Kurihara et al, 2000). This variability in nestin expression levels in the glioma/glioblastoma cell lines caused us to reevaluate nestin expression in human glioma/glioblastomas from low to high malignancy grades. 
For this purpose, we used nestin-specific antiserum and found that vascular endothelial cells contained in glioma tissues express nestin constantly, whether nestin is highly expressed or not in the glial tumor cells. Thus, nestin is a marker protein not only for immature neuroepithelial and glioma cells but also for endothelial cells in active proliferation.

\section{Results}

\section{Nestin Immunostaining for Brain Tumors}

The antibody to nestin was raised in rabbits by injecting the synthetic oligopeptide covering the $\mathrm{C}$-terminal 17 amino acids of the human nestin sequence. This antibody reacted with proteins of 210 to $240 \mathrm{kd}$ from U251 cell extracts by Western blotting, as reported previously (Messam et al, 2000; Tohyama et al, 1992). The immunoblot resembled doublets, perhaps because of the difference in carbohydrate modification, as reported previously (Messam et al, 2000). The immunoblot disappeared when the synthetic oligopeptide was added to the U251 cell lysate, indicating that the immunoblot represents the nestin protein (Fig. 1a).

We further tested the cross-reactivity of this antibody with other intermediate filaments including vimentin, GFAP, keratins, and desmin (Fig. 1b). The antibody to human vimentin showed a single band at a little beyond the 50-kd marker position with both U251 and HeLa cell extracts. The antibody to GFAP displayed an approximately 50-kd band with U251 cell extract, but not with HeLa cell extract. For detecting keratins, we used pooled mouse monoclonal antibodies, anti-cytokeratin AE1/AE3, which recognize a broad subfamily of acidic and basic keratins. The anti-cytokeratin AE1/AE3 recognized an approximately $50-k d$ protein from HeLa cell extract, but faintly from U251 cell extract. The antibody to human desmin did not display bands with either U251 or HeLa cell extract. Nonimmune rabbit serum did not show any artifactual bands with either U251 or HeLa cell extract. Thus, the antibody to nestin displayed large-sized bands corresponding to nestin molecular-sized proteins reported previously (Messam et al, 2000; Tohyama et al, 1992) but did not cross-react with other intermediate filaments (Fig. 1b).

We then immunostained 71 brain tumor samples with this antibody. Table 1 is a summary of the immunostaining. Normal brain cortex tissues were not immunostained with this antibody (Fig. 2, a and b), although a few vascular endothelial cells showed occasional faint staining, as described previously (Dahlstrand et al, 1992). In the glioblastomas (WHO grade IV), the typical nestin staining was fibrillar distribution along the processes of the tumor cells, as shown in Figure 3a. The staining intensity was classified as $4+$ in this tumor. Nestin staining was also evident as a button-like cluster in the cytoplasm of round-shaped tumor cells, as shown in Figure $3 b$ (anaplastic oligoastrocytoma, staining intensity is $3+$ ). In some grade III and grade IV gliomas, the staining
A

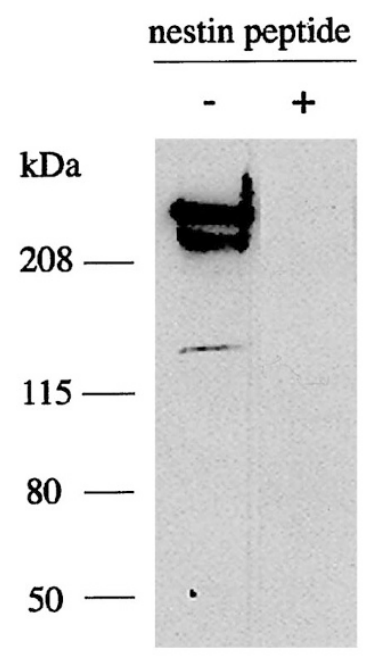

B

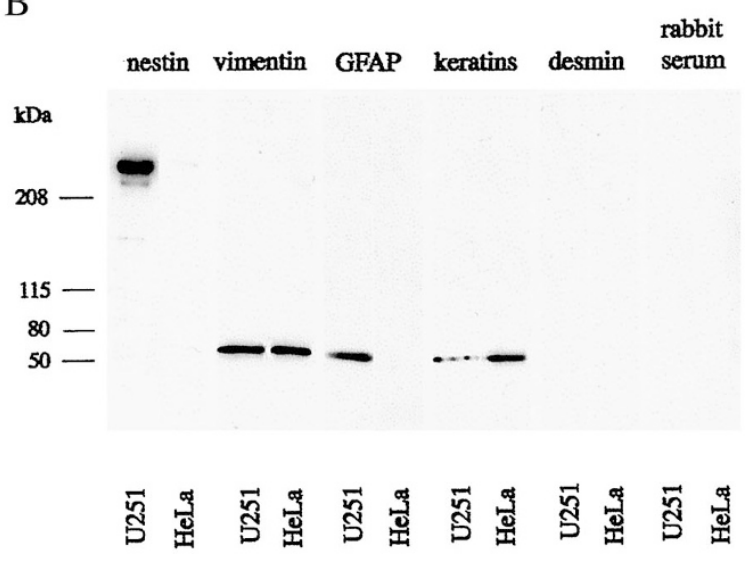

Figure 1.

A, Western blot of nestin. Cell lysates of U251 human glioma cells were run on $7.5 \%$ polyacrylamide gel. After blotting to a nylon membrane, the membrane was immunostained with anti-nestin antibody (1:7500 dilution). Molecular size $(\mathrm{kd})$ is shown to the left of the gel. Left lane, lysate only; right lane, lysate mixed with the synthetic oligopeptide covering $\mathrm{C}$-terminal nestin sequence. B, Western blot of other intermediate filaments, vimentin, glial fibrillary acidic protein (GFAP), keratins, and desmin. Cell lysates of U251 human glioma cells and HeLa human cervix epithelioid cells were used for analysis. Antibodies were used according to the manufacturer's instructions. Six Western blots are shown from left to right, with the antibody to nestin, vimentin, GFAP, keratins, and desmin, and with nonimmune rabbit serum.

was limited to proliferative endothelium, as shown in Figure 3c (glioblastoma, grade IV) and in Figure 3d (anaplastic oligodendroglioma, grade III). In the lowgrade gliomas, the staining was weak to negligible in a considerable number of tumors, but distinct staining was noted along the endothelium in the tumor, as shown in Figure 4, $a$ and $b$ (oligodendroglioma, grade II). This tendency was more marked in other types of brain tumors (Schwannoma, Fig. 4c; and meningioma, Fig. 4d), whose epithelium immunostained strongly positive for nestin while the tumor cells did not stain at all. Thus, the tumor endothelial cells expressed nestin without regard to the WHO grade for malignancy.

\section{Nestin Expression in Replicating Endothelial Cells}

Because tumor epithelium is characterized by neoangiogenic growth (Bergers et al, 2000; Croix et al, 


\begin{tabular}{|c|c|c|c|c|c|c|c|c|c|}
\hline \multirow[b]{3}{*}{ Histology } & \multirow[b]{3}{*}{$n$} & \multicolumn{8}{|c|}{ Intensity of nestin staining } \\
\hline & & \multicolumn{5}{|c|}{ Tumor cells } & \multicolumn{3}{|c|}{ Endothelial cells } \\
\hline & & 0 & $1+$ & $2+$ & $3+$ & $4+$ & 0 & $1+$ & $2+$ \\
\hline \multicolumn{10}{|l|}{ Glioma } \\
\hline \multicolumn{10}{|l|}{ WHO grade I } \\
\hline Pilocytic astrocytoma & 4 & 2 & 1 & 1 & 0 & 0 & 0 & 2 & 2 \\
\hline Subependymoma & 1 & 0 & 0 & 0 & 1 & 0 & 0 & 0 & 1 \\
\hline Ganglioglioma & 1 & 1 & 0 & 0 & 0 & 0 & 0 & 1 & 0 \\
\hline \multicolumn{10}{|l|}{ WHO grade II } \\
\hline Astrocytoma & 3 & 2 & 0 & 1 & 0 & 0 & 0 & 2 & 1 \\
\hline Oligodendroglioma & 1 & 0 & 0 & 0 & 1 & 0 & 0 & 0 & 1 \\
\hline Ependymoma & 4 & 0 & 0 & 3 & 1 & 0 & 0 & 4 & 0 \\
\hline Oligoastrocytoma & 3 & 0 & 0 & 1 & 2 & 0 & 0 & 1 & 2 \\
\hline \multicolumn{10}{|l|}{ WHO grade III } \\
\hline Anaplastic astrocytoma & 7 & 1 & 1 & 4 & 0 & 1 & 1 & 4 & 2 \\
\hline Anaplastic oligodendroglioma & 7 & 1 & 1 & 1 & 4 & 0 & 0 & 1 & 6 \\
\hline Anaplastic ependymoma & 2 & 0 & 0 & 0 & 1 & 1 & 0 & 1 & 1 \\
\hline Anaplastic oligoastrocytoma & 2 & 0 & 0 & 0 & 1 & 1 & 0 & 2 & 0 \\
\hline \multicolumn{10}{|l|}{ WHO grade IV } \\
\hline Glioblastoma & 22 & 2 & 1 & 4 & 8 & 7 & 0 & 12 & 10 \\
\hline \multicolumn{10}{|l|}{ Others } \\
\hline Hemangioblastoma & 4 & * & * & * & * & * & 0 & 0 & 4 \\
\hline Medulloblastoma & 2 & 2 & 0 & 0 & 0 & 0 & 0 & 1 & 1 \\
\hline Atypical teratoid/rhabdoid tumor & 1 & 1 & 0 & 0 & 0 & 0 & 0 & 0 & 1 \\
\hline Meningioma & 3 & 3 & 0 & 0 & 0 & 0 & 0 & 2 & 1 \\
\hline Atypical meningioma & 2 & 2 & 0 & 0 & 0 & 0 & 0 & 0 & 2 \\
\hline Schwannoma & 2 & 0 & 0 & 1 & 1 & 0 & 0 & 0 & 2 \\
\hline Total & 71 & 17 & 4 & 16 & 20 & 10 & 1 & 33 & 37 \\
\hline
\end{tabular}

$n$, sample number.

* In hemangioblastomas, tumor cells are precursors for endothelial cells. To evaluate the nestin immunostaining intensity, we classified hemangioblastoma cells as endothelial cells, but not as tumor cells. Pathologic sections were scanned at low magnification to search for strong nestin-stained areas. Any field containing a large nestin-stained area was then observed with a $10 \times$ objective $(\times 100$ magnification $)$ for classification of the staining intensity.

Definition of nestin immunostaining intensity:

Tumor cells: $\%$ of stained cells over total cell population in one field under $\times 100$ magnification. 0 , no staining; $1+$, less than $1 \%$ of total tumor cell population positive; $2+, 1-5 \%$ of total tumor cell population positive; $3+, 5-30 \%$ of total cell population positive; $4+$, over $30 \%$ of total cell population positive.

Endothelial cells: positive cells found in one microscopic field. 0 , no staining; $1+$, positive cells found by over $\times 200$ magnification; $2+$, positive cells found by $\times 100$ magnification.

2000), we suspected that nestin expression is specific for proliferating endothelial cells. To examine endothelial nestin expression, we used bovine aortic endothelial cells (BAECs). Nestin was expressed strongly in BAECs in a static culture by both Northern blot analysis and immunostaining (Fig. 5, left lane, and Fig. 6a). Endothelial cells proliferate by cell division in static culture, whereas proliferation decreases under physiologic laminar flow (approximately $15 \mathrm{dyn} / \mathrm{cm}^{2}$ ) (Malek et al, 1999). To examine whether the nestin expression is proliferation dependent, BAECs were subjected to a shear stress flow of $15 \mathrm{dyn} / \mathrm{cm}^{2}$ for 12 hours. Expression of nestin mRNA diminished significantly with shear stress flow by Northern blot analysis (Fig. 5, right lane). Furthermore, we confirmed a flowdependent decrease of nestin expression by immunostaining (Fig. 6b).

\section{Nestin Expression in Hemangioblastomas}

Because nestin was expressed in proliferating endothelial cells, we suspected that it is expressed even in hemangioblastomas because hemangioblasts are thought to be a precursor for both hematopoietic cells and angioblasts (Eichmann et al, 1997). To examine this issue, we tested four human hemangioblastomas (Table 1). An endothelial cell marker, von Willebrand factor, positively immunostained along endothelium and microcapillary vessels in the hemangioblastoma (Fig. 7b) (Böhling et al, 2000). Nestin also immunostained mostly in microcapillary vessels in the hemangioblastoma (Fig. 7c). However, typical endothelium consisting of thin cytoplasm with a convex-shaped nucleus was not positive for nestin staining (red arrows in Fig. 7c). Because the appearance of endothelium is similar to normal vessels, this type of endothelium may have been well-differentiated and lost nestin expression. Nestin-positive cells consisting of hemangioblastomas may reflect genuine transformed hemangioblasts. Thus, nestin is a marker protein not only for neuroepithelial stem cells and glioma cells but also for hemangioblasts and proliferating endothelial cells. 


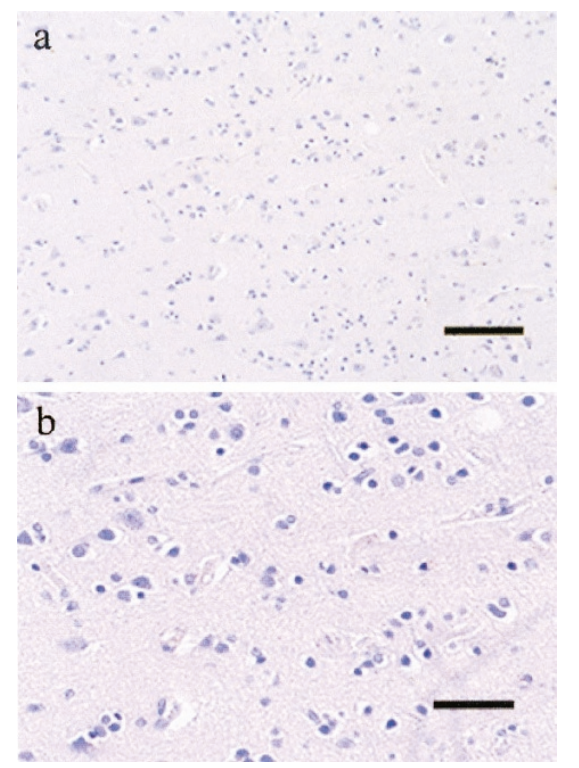

Figure 2.

Nestin immunostaining of control human brain tissues. a, human cortex. Scale, $200 \mu \mathrm{m}$. b, close-up of a. Scale, $50 \mu \mathrm{m}$.

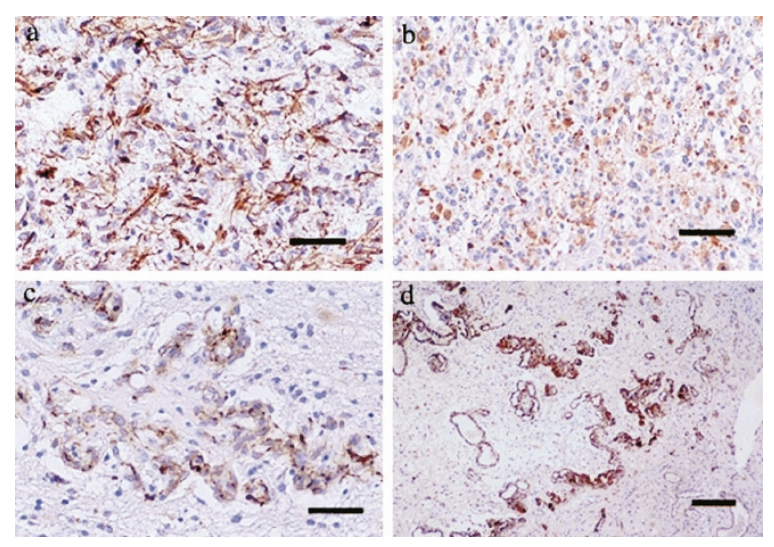

Figure 3.

Nestin immunostaining of WHO high-grade malignancy gliomas. a, Grade IV glioblastoma. The intensity of the nestin staining is $4+$. b, Grade III anaplastic oligoastrocytoma. The intensity is $3+$. c, Grade IV glioblastoma. The staining is limited to the proliferative endothelium. The staining intensity of the endothelium is $2+$. d, Grade III anaplastic oligodendroglioma. The staining is also limited to the proliferative endothelium. The staining intensity of the endothelium is $2+$. Scales, $50 \mu \mathrm{m}$ (a to c) and $200 \mu \mathrm{m}$ (d).

\section{Discussion}

The present study demonstrated that the neuroepithelial stem cell-specific marker nestin filament is expressed distinctly in the vascular endothelium draining brain tumors. The nestin immunostaining was distinct in tumor endothelium but faint to negligible in brain tumor cells (Fig. 3, c and d, and Fig. 4, a to d). Proliferating endothelial cells during active angiogenesis for tumor expansion appear to express the nestin filament. This expression may not be specific for tumor-associated angiogenesis because bovine endothelial cells in culture express nestin to a great extent. Physiologic level of laminar shear stress flow (approximately $15 \mathrm{dyn} / \mathrm{cm}^{2}$ ) induces endothelial qui-
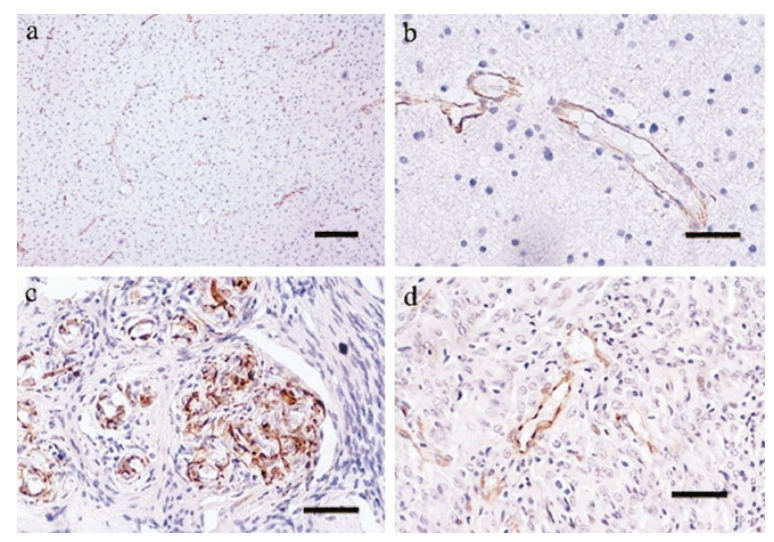

Figure 4.

Nestin immunostaining of WHO low-grade malignancy gliomas and other types of brain tumors. Nestin staining is not found in tumor cells but is instead limited to the proliferative endothelium. a, Grade II oligodendroglioma. The staining intensity of the endothelium is $2+$. b. High magnification of a. c, Schwannoma. The staining intensity is $2+$. d, Meningioma. The staining intensity is $2+$. Scales, $200 \mu \mathrm{m}$ (a) and $50 \mu \mathrm{m}$ (b to d).

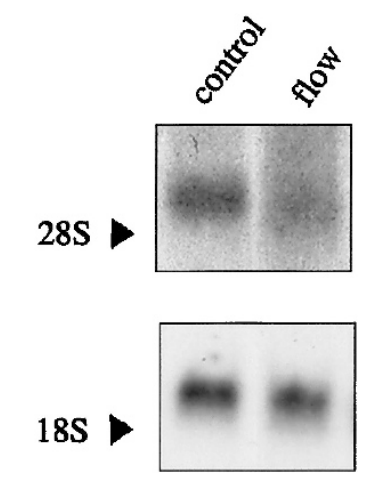

Figure 5.

Expression of nestin mRNA in shear stress-loaded endothelial cells. Upper panel, nestin mRNA blot: left lane, RNA from bovine aortic endothelial cells (BAECs) in static culture; right lane, RNA from shear stress-loaded BAECs for 12 hours. $28 \mathrm{~S}$ indicates ribosomal RNA size. Lower panel, glyceraldehyde-3phosphate dehydrogenase mRNA blot as a control: right and left lanes are the same as those in the upper panel. 18S indicates ribosomal RNA size.

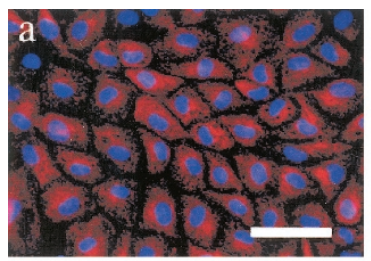

control

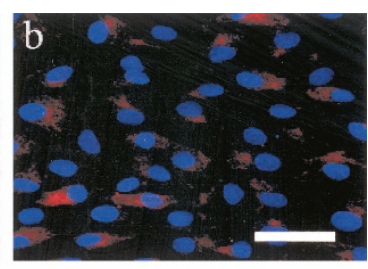

flow

\section{Figure 6.}

Nestin immunostaining in shear stress-loaded endothelial cells. a, BAECs in static culture. b, BAECs under shear stress load $\left(15 \mathrm{dyn} / \mathrm{cm}^{2}\right)$ for 12 hours. Scales, $50 \mu \mathrm{m}(\mathrm{a}$ and $\mathrm{b})$.

escence and an atheroprotective gene expression, whereas low or turbulent shear stress flow stimulates the expression of proinflammatory and atherogenic genes (Gimbrone et al, 1997; Malek et al, 1999). When the physiologic level of shear stress flow was loaded to endothelial cells, nestin gene expression decreased 


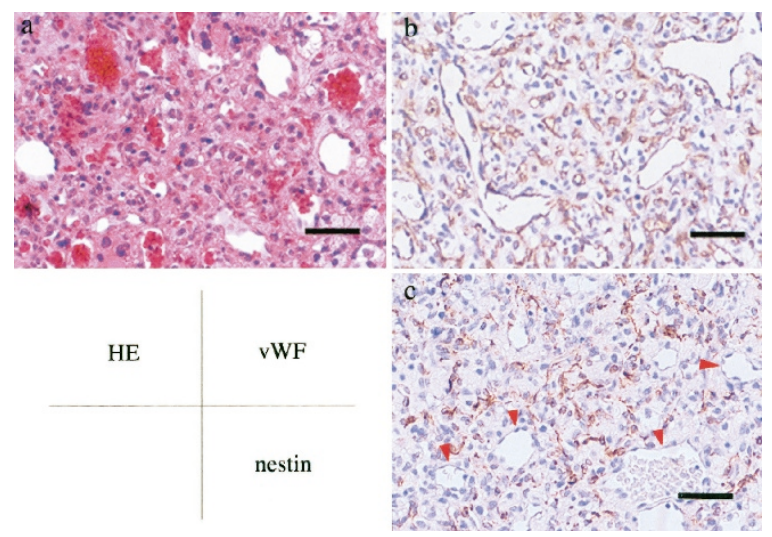

Figure 7.

Staining of hemangioblastomas. a, Hematoxylin and eosin staining. b, Immunostaining for von Willebrand factor. c, Immunostaining for nestin. Red arrows, nonstained endothelium. Scales, $50 \mu \mathrm{m}$ (a to $\mathrm{C}$ ). $\mathrm{HE}=$ hematoxylin and eosin; vWF $=$ von Willebrand factor.

remarkably, suggesting that the nestin expression is specific for proliferating endothelial cells. Thus, nestin serves as a marker for proliferating endothelial cells, especially for angiogenic endothelium draining brain tumor tissues.

Nestin was initially found in growing neuroepithelial stem cells and gliomas and has been recognized as an oncofetal marker protein for gliomas (Dahlstrand et al, 1992; Lendahl et al, 1990). When nestin expression was further investigated in detail by nestin promoter/ intron-driven LacZ expression in mice, the expression was found to be limited to neuroepithelial stem cells and somites in developing mouse embryos (Zimmerman et al, 1994). This neuroepithelial stem cell-specific expression was shown to be driven by the second intron of the nestin gene, whereas the somite-specific expression seemed to be promoted by the first intron. Although nestin immunostaining has been reported to be weak in a few vascular endothelial cells in normal brain tissues in rats and humans (Dahlstrand et al, 1992; Tohyama et al, 1992, 1993), the endotheliumspecific expression of the nestin gene remains to be investigated. Because both somite and endothelium originate from the embryonal mesoderm, it should be investigated whether endothelium-specific nestin expression is driven by the first intron.

The extensive nestin immunostaining in hemangioblastomas is of interest because dysfunction of the tumor suppressor gene von Hippel-Lindau (pVHL) predisposes towards the development of central nervous system hemangioblastomas (Böhling et al, 2000). $\mathrm{pVHL}$ functions as a component of the proteasome complex for degrading hypoxia-inducible factors (HIF) (Maxwell et al, 1999). Although HIF-1 is easily inducible by hypoxia, HIF-1 is readily degraded by ubiquitination in a pVHL-dependent fashion in normal vascular stromal cells (Ohh et al, 2000). However, dysfunction of pVHL by genetic defects results in an increase in HIF-1 because of lack of its degradation system. Increased HIF-1 molecules induce vascular endothelial growth factor, which in turn activates its receptor Flk-1 and Flt-1 on endothelial plasma mem- branes, resulting in active angiogenesis (Dang and Semenza, 1999). Endothelial cells express HIF-like factor (HLF, or EPAS1), which is also induced by hypoxia and is degraded by the pVHL degradation system (Ema et al, 1997; Krieg et al, 2000). HIF-1 and HLF are known to act on its consensus enhancer element 5'-CACGTG-3'. We looked for this element on the first and second introns of the nestin gene using a computer search and found at least one candidate site on the first intron. It will be important to examine whether this candidate site is functional for the HIF/ HLF-dependent expression of the nestin gene in tumor endothelium.

Although a number of angiogenesis-related genes are reported in colorectal cancer endothelium, the nestin gene is not included in the list (Croix et al, 2000). Angiogenesis-related genes in brain tumor endothelium may be different from those in colorectal endothelium. It is noteworthy that strong nestin expression is found in brain tumor endothelium even if no nestin expression is found in the brain tumor cells. We suggest that nestin serves as an excellent endothelium marker for brain tumors such as gliomas, hemangioblastomas, Schwannomas, medulloblastomas, and meningiomas.

\section{Materials and Methods}

\section{Preparation and Characterization of Nestin Antibody}

The antibody to human nestin was generated in rabbits using the synthetic oligopeptide covering the C-terminal 17 amino acids: 1602-KFTQREGDRESWSSGED-1618 (Messam et al, 2000). A cysteine residue was added to the N-terminal of this oligopeptide for covalent conjugation with the carrier protein keyhole limpet hemocyanin. The oligopeptide-protein conjugate was emulsified with Freund's complete adjuvant and injected into two New Zealand White rabbits. After several booster injections, the rabbits developed antibodies against the oligopeptide.

The specificity of the antibody was examined by Western blot analysis using cell extracts from a human glioblastoma cell line U251 and from a human cervix epithelioid cell line HeLa. For testing cross-reactivity of the nestin antibody with other intermediate filaments, we used four antibodies recognizing human vimentin, human GFAP, human keratins, and human desmin. Antibody to GFAP was made previously by one of the coauthors, Dr. Nakazato (Nakazato et al, 1982). Other antibodies were purchased from DAKO, Glostrup, Denmark.

\section{Morphologic Studies}

We used 71 human brain tumor samples including 57 gliomas and 14 other brain tumors. The gliomas included 6 WHO grade I tumors, 11 grade II tumors, 18 grade III tumors, and 22 grade IV tumors, as listed in Table 1. Other brain tumors included four hemangioblastomas, two medulloblastomas, one atypical teratoid/rhabdoid tumor, three meningiomas, two atypical meningiomas, and two Schwannomas. All 71 brain tumors were resected at the Department of Neurosur- 
gery, Gunma University School of Medicine. The diagnoses were established by routine pathologic examinations according to the revised WHO classification at the Department of Pathology, Gunma University School of Medicine.

The human brain and tumor tissues and BAECs were fixed in $4 \%$ paraformaldehyde in $0.1 \mathrm{M}$ phosphate buffer, $\mathrm{pH} 7.4$, for 24 hours (tissues) or 1 hour (culture cells). Small pieces of the tissue sample were embedded in optimal cutting temperature compound for microtome sectioning. BAECs were treated with 50 $\mathrm{mM} \mathrm{NH}_{4} \mathrm{CL}$ in PBS to quench any free aldehyde, then made permeable by $0.1 \%$ saponin and $0.4 \%$ bovine serum albumin before the primary antibody incubation. The tissue sections or BAECs were first incubated with the primary antibody to nestin at a dilution of 1:5000. For brain and tumor tissues, an LSAB2/HRP staining kit (DAKO) was used as the secondary antibody reaction system. The procedure consists of a secondary antibody reaction followed by an enzyme reaction with a horseradish peroxidase-labeled streptavidin system. In the enzyme reaction, the peroxidase catalyzes 3-amino-9-ethylcarbazole to an insoluble brown-colored product. For BAECs, the secondary antibody used was indodicarbocyanideconjugated affinity-purified donkey anti-rabbit lgG (red colored) (Jackson ImmunoResearch, West Grove, Pennsylvania). The nucleus was counterstained blue with 4,6-diamidino-2-phenylindole.

\section{Shear Stress Flow Experiment}

We used BAECs that were scraped off the inner surface of the bovine thoracic aortas using a razor blade. The BAECs were then cultured in 6-well plates in RPMI 1640 with $20 \%$ fetal bovine serum. When BAEC cells formed a colony of 3 to $6 \mathrm{~mm}$ in diameter, the cells were moved to new 6 -well plates, where fetal bovine serum was decreased to $10 \%$. Culture cell lines growing in a cobble stone-like sheet formation were selected by 7 to 12 passages and stored in liquid nitrogen until use.

BAECs were cultured on 0.5-mm-thick quartz cover glass. The cover glass was inverted and placed on a parallel plate-type flow chamber (inner space size: 16 $\mathrm{mm}$ wide $\times 35 \mathrm{~mm}$ long $\times 200 \mu \mathrm{m}$ deep), as described previously (Negishi et al, 2001). The apparatus was placed in a $\mathrm{CO}_{2}$ incubator at $37^{\circ} \mathrm{C}$. The shear stress forces were calculated based on an equation described previously (Negishi et al, 2001). The flow rate was adjusted to $15 \mathrm{dyn} / \mathrm{cm}^{2}$, which is comparable to the physiologic flow rate.

\section{Northern and Western Blot Analyses}

For Northern blot analysis, total RNA was extracted from BAECs, denatured with $6.3 \%$ formaldehyde $/ 50 \%$ formamide, electrophoresed on a $1.0 \%$ agarose gel containing $6.6 \%$ formaldehyde, then blotted to a nylon membrane (Amersham Life Science, Tokyo, Japan). Hybridization was performed with a probe of human nestin DNA fragment (560 bp), labeled with $\alpha^{-32} \mathrm{P}$
deoxy-CTP by a random priming procedure. A glyceraldehyde-3-phosphate dehydrogenase probe was used as a control.

For Western blotting, U251 human glioblastoma cells were solubilized for cell lysates in lysis buffer (70 $\mathrm{mm}$ Tris- $\mathrm{HCl}$, pH 6.8, 11.2\% glycerol, 3\% SDS, 0.01\% bromophenol blue, 5\% 2-mercaptoethanol). The cell lysates were then subjected to electrophoresis on a $7.5 \%$ polyacrylamide gel under a reducing condition, then blotted onto a nitrocellulose membrane for probing with rabbit anti-human nestin antiserum at a dilution of 1:7500. Nestin blots were detected utilizing an ECL detection system (Amersham, Buckinghamshire, United Kingdom).

\section{Acknowledgements}

We thank Ms. Eiko Hamana for her secretarial assistance and Ms. Mari Hosoi for her technical assistance.

\section{References}

Bergers G, Brekken R, McMahon G, Vu TH, Itoh T, Tamaki K, Tanzawa K, Thorpe P, Itohara S, Werb Z, and Hanahan D (2000). Matrix metalloproteinase-9 triggers the angiogenic switch during carcinogenesis. Nat Cell Biol 2:737-744.

Böhling T, Plate KH, Haltia MJ, Alitalo K, and Neumann HPH (2000). Von Hippel-Lindau disease and capillary haemangioblastoma. In: Kleihues P and Cavenees WK, editors. Pathology and genetics of tumours of the nervous system. Lyon: IARC Press, 223-226.

Croix BS, Rago C, Velculescu V, Traverso G, Romans KE, Montgomery E, Lal A, Riggins GJ, Lengauer C, Vogelstein B, and Kinzler KW (2000). Genes expressed in human tumor endothelium. Science 289:1197-1202.

Dahlstrand J, Collins VP, and Lendahl U (1992). Expression of the class $\mathrm{VI}$ intermediate filament nestin in human central nervous system tumors. Cancer Res 52:5334-5341.

Dang CV and Semenza GL (1999). Oncogenic alterations of metabolism. Trends Biochem Sci 24:68-72.

Eichmann A, Corbel C, Nataf V, Vaigot P, Bréant C, and Douarin NML (1997). Ligand-dependent development of the endothelial and hemopoietic lineages from embryonic mesodermal cells expressing vascular endothelial growth factor receptor 2. Proc Natl Acad Sci USA 94:5141-5146.

Eliasson C, Sahlgren C, Berthold C-H, Stakeberg J, Celis JE, Betsholtz C, Eriksson JE, and Pekny M (1999). Intermediate filament protein partnership in astrocytes. J Biol Chem 274: 23996-24006.

Ema M, Toya S, Yokotani N, Sogawa K, Matsuda Y, and Fujii-Kuriyama $Y$ (1997). A novel bHLH-PAS factor with close sequence similarity to hypoxia-inducible factor $1 \alpha$ regulates the VEGF expression and is potentially involved in lung and vascular development. Proc Natl Acad Sci USA 94:42734278.

Gimbrone MA Jr, Nagel T, and Topper JN (1997). Biomechanical activation: An emerging paradigm in endothelial adhesion biology. J Clin Invest 99:1809-1813.

Krieg M, Haas R, Brauch H, Acker T, Flamme I, and Plate KH (2000). Up-regulation of hypoxia-inducible factors HIF-1 $\alpha$ and HIF-2 $\alpha$ under normoxic conditions in renal carcinoma 
cells by von Hippel-Lindau tumor suppressor gene loss of function. Oncogene 19:5435-5443.

Kurihara H, Zama A, Tamura M, Takeda J, Sasaki T, and Takeuchi T (2000). Glioma/glioblastoma-specific adenoviral gene expression using the nestin gene regulator. Gene Ther 7:686-693.

Lendahl U, Zimmerman LB, and Mckay RDG (1990). CNS stem cells express a new class of intermediate filament protein. Cell 60:585-595.

Lothian C and Lendahl U (1997). An evolutionarily conserved region in the second intron of the human nestin gene directs gene expression to CNS progenitor cells and to early neural crest cells. Eur J Neurosci 9:452-462.

Malek AM, Alper SL, and Izumo S (1999). Hemodynamic shear stress and its role in atherosclerosis. JAMA 282:20352042.

Maxwell PH, Wiesener MS, Chang G-W, Clifford SC, Vaux EC, Cockman ME, Wykoff CC, Pugh CW, Maher ER, and Ratcliffe PJ (1999). The tumour suppressor protein VHL targets hypoxia-inducible factors for oxygen-dependent proteolysis. Nature 399:271-275.

Messam CA, Hou J, and Major EO (2000). Coexpression of nestin in neural and glial cells in the developing human CNS defined by a human-specific anti-nestin antibody. Exp Neurol 161:585-596.

Nakazato Y, Ishizeki J, Takahashi K, Yamaguchi H, Kamei T, and Mori T (1982). Localization of S-100 protein and glial fibrillary acidic protein-related antigen in pleomorphic adenoma of the salivary glands. Lab Invest 46:621-626.
Negishi M, Lu D, Zhang Y-Q, Sawada Y, Sakai T, Kayo T, Ando J, Izumi T, Kurabayashi M, Kojima I, Masuda H, and Takeuchi T (2001). Upregulatory expression of furin and transforming growth factor- $\beta$ by fluid shear stress in vascular endothelial cells. Arterioscler Thromb Vasc Biol 21:785-790.

Ohh M, Park CW, Ivan M, Hoffman MA, Kim T-Y, Huang LE, Pavletich N, Chau V, and Kaelin WG (2000). Ubiquitination of hypoxia-inducible factor requires direct binding to the $\beta$-domain of the von Hippel-Lindau protein. Nat Cell Biol 2:423-427.

Rutka JT, Ivanchuk S, Mondal S, Taylor M, Sakai K, Dirks P, Jun P, Jung S, Becker LE, and Ackerley C (1999). Coexpression of nestin and vimentin intermediate filaments in invasive human astrocytoma cells. Int $\mathrm{J}$ Dev Neurosci 17: 503-515.

Tohyama T, Lee VM-Y, Rorke LB, Marvin M, McKay RDG, and Trojanowski JQ (1992). Nestin expression in embryonic human neuroepithelium and in human neuroepithelial tumor cells. Lab Invest 66:303-313.

Tohyama T, Lee VM-Y, Rorke LB, Marvin M, McKay RDG, and Trojanowski JQ (1993). Monoclonal antibodies to a rat nestin fusion protein recognize a $220-\mathrm{kDa}$ polypeptide in subsets of fetal and adult human central nervous system neurons and in primitive neuroectodermal tumor cells. Am J Pathol 143:258-268.

Zimmerman L, Lendahl U, Cunningham M, Mckay R, Parr B, Gavin B, Mann J, Vassileva G, and McMahon A (1994). Independent regulatory elements in the nestin gene direct transgene expression to neural stem cells or muscle precursors. Neuron 12:11-24. 